\title{
Genotype-phenotype map characteristics of an in silico heart cell
}

\section{Jon Olav Vik ${ }^{1}{ }^{*}$, Arne B. Gjuvsland ${ }^{1}$, Liren Li ${ }^{2}$, Kristin Tøndel ${ }^{1}$, Steven Niederer ${ }^{3}$, Nicolas P. Smith ${ }^{2,3}$, Peter J. Hunter ${ }^{4}$ and Stig W. Omholt ${ }^{5}$}

${ }^{1}$ Department of Mathematical Sciences and Technology, Centre for Integrative Genetics, Norwegian University of Life Sciences, Ås, Norway

2 Department of Computer Science, University of Oxford, Oxford, UK

${ }^{3}$ Department of Biomedical Engineering, St. Thomas' Hospital, King's College London, London, UK

${ }^{4}$ Auckland Bioengineering Institute, The University of Auckland, Auckland, New Zealand

${ }^{5}$ Department of Animal and Aquacultural Sciences, Centre for Integrative Genetics, Norwegian University of Life Sciences, Ås, Norway

\section{Edited by:}

Joseph Nadeau, Case Western

Reserve University, USA

Reviewed by:

Howard Prentice, Florida Atlantic University, USA

Rachael Hageman Blair, University at Buffalo, USA

${ }^{*}$ Correspondence:

Jon Olav Vik, Department of Mathematical Sciences and

Technology, Centre for Integrative

Genetics, Norwegian University of

Life Sciences, P.O. Box 5003, N-1432

Ås, Norway.

e-mail:jonovik@gmail.com
Understanding the causal chain from genotypic to phenotypic variation is a tremendous challenge with huge implications for personalized medicine. Here we argue that linking computational physiology to genetic concepts, methodology, and data provides a new framework for this endeavor. We exemplify this causally cohesive genotype-phenotype (cGP) modeling approach using a detailed mathematical model of a heart cell. In silico genetic variation is mapped to parametric variation, which propagates through the physiological model to generate multivariate phenotypes for the action potential and calcium transient under regular pacing, and ion currents under voltage clamping. The resulting genotype-to-phenotype map is characterized using standard quantitative genetic methods and novel applications of high-dimensional data analysis. These analyses reveal many well-known genetic phenomena like intralocus dominance, interlocus epistasis, and varying degrees of phenotypic correlation. In particular, we observe penetrance features such as the masking/release of genetic variation, so that without any change in the regulatory anatomy of the model, traits may appear monogenic, oligogenic, or polygenic depending on which genotypic variation is actually present in the data. The results suggest that a cGP modeling approach may pave the way for a computational physiological genomics capable of generating biological insight about the genotype-phenotype relation in ways that statistical-genetic approaches cannot.

Keywords: causally cohesive genotype-phenotype modeling, multivariate genotype-to-phenotype map, cGP heart model, penetrance, epistasis

\section{INTRODUCTION}

A comprehensive understanding of how genetic variation causes phenotypic variation of a complex trait is a long-term disciplinary goal of genetics (Bateson, 1906). The idea of linking system dynamics and genetics dates back to Burns (1970) and has proved fruitful in relatively simple cases (Omholt et al., 2000; Gilchrist and Nijhout, 2001; Peccoud et al., 2004; Welch et al., 2005; Gjuvsland et al., 2007a,b,c, 2010; Rajasingh et al., 2008; Martens et al., 2009). The basic premise is that in a well-validated model that is capable of accounting for the phenotypic variation in a population, the causative genetic variation will manifest in the model parameters. In this context, the term "phenotype" refers to any relevant measure of model behavior, whereas the term "parameter" denotes a quantity that is constant over the time-scale of the particular model being studied. However, model parameters are themselves phenotypes (Rajasingh et al., 2008), whose genetic basis may be mono-, oligo-, or polygenic, and whose physiological basis can be mechanistically modeled at ever deeper levels of detail. The term causally cohesive genotype-phenotype (cGP) modeling (Rajasingh et al., 2008) thus denotes an approach where low-level parameters have an articulated relationship to the individual's genotype, and higher-level phenotypes emerge from the mathematical model describing the causal dynamic relationships between these lower-level processes. It aims to bridge the gap between standard population genetic models where the connection between genotypes and phenotypes is described in the form of "genotypic values," i.e., the expected phenotypic value for a given genotype (Falconer and Mackay, 1996; Lynch and Walsh, 1998), and mechanistic physiological models without an explicit genetic basis. This forces a causally coherent depiction of the genotype-to-phenotype (GP) map.

Computational physiology is the natural habitat for this endeavor. Computational modeling of multiscale physiology is intimately tied to experimental studies and has offered unique and generic insights into medically important mechanisms (Noble, 2002b). Mechanistic models facilitate confrontation with empirical data, incorporating conservation of charge, mass and momentum, and other physical laws that serve to constrain the mapping from low-level parameters to higher-level phenotypes (Hunter and Borg, 2003). Dealing with genetic and other individual differences is a key next step (Hunter et al., 2010), which will require the incorporation of genotypic data and the application 
of multivariate numerical and statistical methods in computational physiology. The discipline thus defined may be called computational physiological genomics.

The mammalian heart is arguably the best available cGP model system, being studied in detail at levels from protein subunits through ion channels and calcium handling, cellular action potential (time-course of transmembrane voltage, $V$ ) and calcium transient (time-course of cytosolic $\mathrm{Ca}^{2+}$ concentration, Cai), tissue electrophysiology, mechanics, and fluid dynamics (Noble, 2002a). Much of this research has been medically motivated, as anomalies in these processes can give rise to disease in a complex interplay between genetic, age-related, and lifestyle factors. However, analyzing whole-organ models is computationally and conceptually challenging, and many submodels are of quite intimidating complexity in themselves.

Here, as a pilot for a whole-organ cGP study, we explore, characterize, and analyze a detailed model of a mouse heart cell ( $\mathrm{Li}$ et al., 2010), built to account for the action potential (electrical signal) and calcium transient (linked to muscle contraction) of the cardiomyocyte in terms of its constituent ion currents and gating channels. Cardiac ion channels are prime candidates for realistic gene-to-parameter mapping, being quite low-level parameters whose genetic variation has been well studied (Roberts and Brugada, 2003; Roepke and Abbott, 2006; Sanguinetti and TristaniFirouzi, 2006). Under the assumption that genetic variation manifests in low-level parameters, simulations of the model generate in silico high-dimensional phenotypes, ranging from individual ion currents to the action potential and calcium transient.

By use of the single heart cell cGP model we show (1) how the statistical-genetic architecture of traits may arise, (2) how multivariate analysis methods can be used to extract information about high-dimensional GP maps created by cGP models, (3) how the cGP framework can be used to identify genetic variation underlying disease phenotypes, and (4) how the cGP framework can be used to systematically disclose how the genetic background may affect penetrance, i.e., the proportion of affected individuals among those carrying a predisposing allele. The paper thus addresses several key disciplinary aspects of physiological genomics, and it exemplifies many of the methodological challenges pertaining to whole-organ models, while being computationally inexpensive enough to allow a more exhaustive exploration.

\section{METHODS \\ HEART CELL MODEL}

The LNCS cell model (Li et al., 2010) extends that of Bondarenko et al. (2004) with more realistic calcium handling, detailed reparameterization to consistent experimental data, and consistency checking by conservation of charge (cf. Hund et al., 2001). State variables include concentrations of sodium, potassium, and calcium in the cytosol, calcium concentration in the sarcoplasmic reticulum, and the state distribution of ion channels, whose transition rates between open, closed, and inactivated conformations may depend on transmembrane voltage. A simplified overview is given in Figure 1. The model is available as Supplementary Material in CellML and PDF formats. (For details, see Bondarenko et al., 2004; Li et al., 2010) Whereas many cell models are built

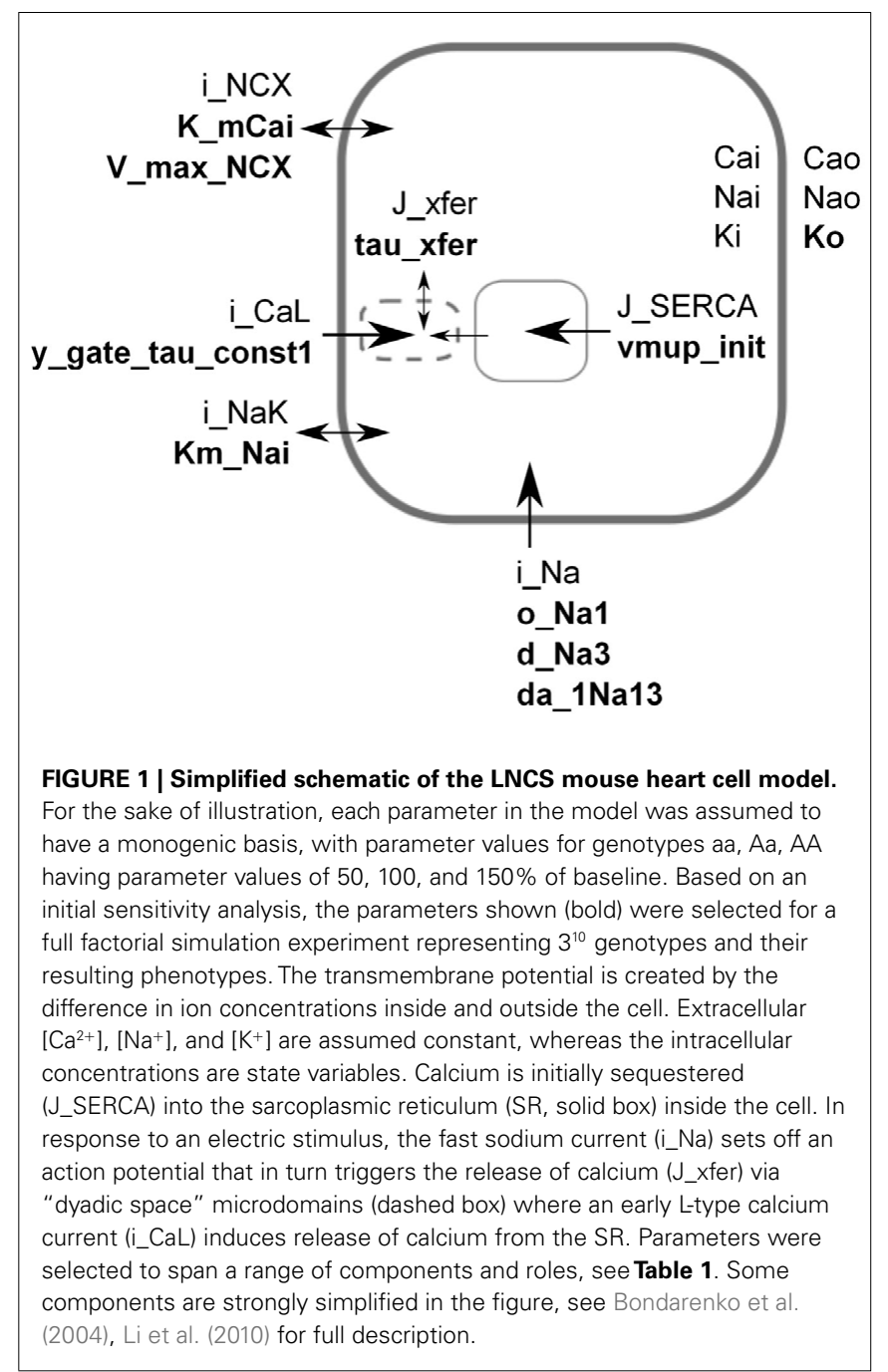

from heterogeneous data sets that span species and temperature (Niederer et al., 2008), essential parts of the LNCS model have been directly fitted to a consistent experimental data set for the C57BL/6 "black 6" mouse, a popular strain for genetic manipulation in studying cardiac electrophysiology and the regulation of intracellular calcium transport. Formulated as a system of 35 coupled ordinary differential equations with 175 parameters (see Unhardcoding of Parameters below), this model provides a comprehensive representation of membrane-bound channels and transporter functions as well as fluxes between the cytosol and intracellular organelles. Below, the term "baseline" refers to the point estimate for the parameter values of the LNCS model, and phenotypes arising from simulations with the baseline parameter scenario.

\section{VIRTUAL EXPERIMENTS AND PHENOTYPES}

We studied phenotypes defined by four experimental protocols described in Bondarenko et al. (2004). Voltage-clamp protocols induce series of stepwise changes in transmembrane voltage (items 3 and 4 below) that are designed to characterize the voltagedependent conformation switching behavior and "memory" of 
ion channels (Molleman, 2002), offering a common basis for comparing the ion-channel behavior of different cell types, models, or parameter scenarios. The protocols were:

1. No stimulus, yielding the quiescent cell state as a phenotype.

2. Regular pacing from quiescence to steady-state dynamics or alternans (action potentials of alternating amplitude), implemented as an external stimulus current of $\mathrm{K}^{+}$ions. Raw phenotypes were the multivariate time-series of state variables during a steady-state action potential (or series of action potentials in the case of alternans), as well as important terms in the rates of change, such as ion currents. The main cell-level phenotypes are the action potential (electrical signal) and calcium transient (linked to muscle contraction), i.e., the time-courses of the transmembrane potential and cytosolic calcium concentration, respectively. Aggregate measures for these phenotypes include action potential duration to $90 \%$ repolarization (APD90), similar measures for 25,50 , and 75\% repolarization, and action potential amplitude and time to peak (Figure 2). APD decay rate, $\lambda$, was computed from the exponential approximation $V(t)=V(0) \exp (-\lambda t)$ based on voltage and time from 50 to $90 \%$ repolarization. Analogous measures were used for the calcium transient.

A stimulus current of $-15 \mathrm{~V} / \mathrm{s}$ was applied for $3 \mathrm{~ms}$ at the start of each stimulus interval. This was repeated until convergence or a maximum of $10 \mathrm{~min}$ simulated model time. Convergence was checked by comparing successive intervals with respect to initial values of each state variable, as well as the integral of the state variable's trajectory over that interval. A running history of 10 intervals was kept, and after each interval we checked for a match (within a relative tolerance of $1 \%$ for all state variables) against the previous ones. This was done for stimulus intervals of 100,200 , and $300 \mathrm{~ms}$.

3. Double-pulse voltage-clamp protocol to estimate the rate and voltage-dependence of ion-channel inactivation. The simulated cell is initially kept at a low holding potential $V_{\text {hold }}$, followed by an abrupt increase to some voltage $V_{1}$ for duration $t_{1}$ (pulse $\mathrm{P} 1$ ), then set to some voltage $V_{2}$ (pulse P2). The main experimental parameters are the duration and voltage of pulse 1 . Raw phenotypes are the same as for regular pacing, with emphasis on the total current (e.g., i_Na for the fast sodium current) and the

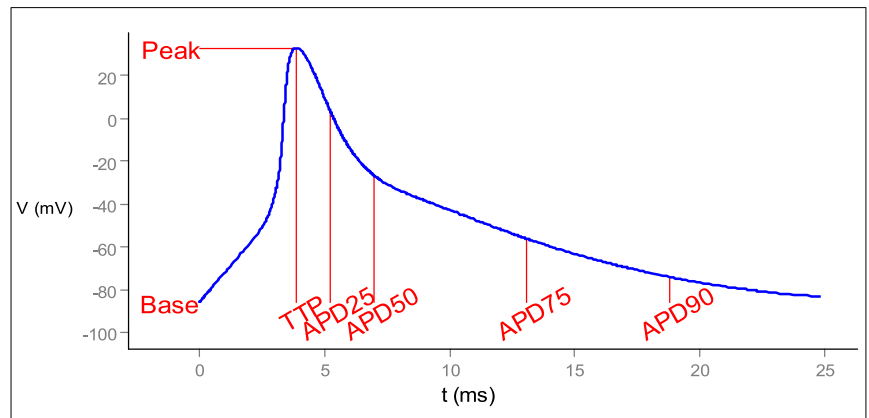

FIGURE 2 | Scalar measures of state-variable trajectories, exemplified by the action potential. TTP, time to peak. APD25, etc.: action potential duration to $25 \%$, etc., return to initial ("base") value. proportion of ion channels in each conformation. Aggregate phenotypes include peak P1 current and the "rate of inactivation" $\tau$, describing the roughly exponential decline in current during the first pulse (P1) as:

$I(t)=I_{0} \exp \left(-\frac{t}{\tau}\right)$,

and fitted to the interval from 95 to $5 \%$ of the peak current.

4. Variable-gap voltage-clamp protocol to estimate the rate and voltage-dependence of recovery from inactivation. From the holding potential, an initial depolarizing pulse P1 will inactivate a high proportion of the ion channels, and is followed by a repolarizing interpulse interval of variable duration (the main experimental parameter), then another pulse P2. Current magnitude during P2 measures recovery from inactivation as a function of interpulse duration. The results were summarized by $I_{\text {peak,max }}$ and $t_{\text {half }}$ in the equation

$$
I_{\text {peak }}\left(t_{g}\right)=I_{\text {peak, max }} \frac{t_{g}}{t_{g}+t_{\text {half }}},
$$

where $t_{g}$ is the gap duration and $I_{\text {peak }}$ is the peak P2 current.

\section{DISEASE PHENOTYPE}

Cell dynamics was categorized as "failed" based on the calcium transient if the peak was below $50 \%$ of baseline (illustrating failure to contract), if the base was more than $200 \%$ of baseline (failure to relax), if amplitude was less than $50 \%$ of baseline (at $200 \mathrm{~ms}$ pacing), or if dynamics failed to converge within $10 \mathrm{~min}$ of simulated time. Details of alternans were not pursued in this paper.

\section{UNHARDCODING OF PARAMETERS}

Many, if not most, published cell models include constants that are arguably better viewed as parameters for the purpose of cGP studies, such as the voltage-sensitivity of ion-channel behavior. For example, the sodium channel of the Bondarenko model contains just one parameter but 28 hardcoded constants (Bondarenko et al., 2004, Eq. A51-A64). We used the Python package lxml 2.3 ${ }^{1}$ to scan a CellML (Lloyd et al., 2004) representation of the LNCS model for constants, except for physical constants (such as the Faraday constant) and ion charges (e.g., 2 for $\mathrm{Ca}^{2+}$ ). This brought the number of parameters up from 73 to 175 , not counting physical constants or parameters relating to pacing protocols.

\section{LOCAL SENSITIVITY ANALYSIS}

For a local overview of the genotype-to-phenotype map, we estimated the first derivatives of scalar phenotype measurements $\phi_{\mathrm{k}}$ with respect to model parameters $p_{\mathrm{i}}$, using central differences with a $10 \%$ step size. For parameters and phenotypes that have a non-arbitrary zero point, it is meaningful to scale the first derivatives into elasticities, i.e., dimensionless ratios of relative changes. This is equivalent to log-transforming the quantities before taking derivatives.

$e_{\mathrm{ik}}=\frac{d \phi_{\mathrm{k}} / \phi_{\mathrm{k}}}{d p_{\mathrm{i}} / p_{\mathrm{i}}}=\frac{d \phi_{\mathrm{k}}}{d p_{\mathrm{i}}} \frac{p_{\mathrm{i}}}{\phi_{\mathrm{k}}}=\frac{d \ln \phi_{\mathrm{k}}}{d \ln p_{\mathrm{i}}}$

${ }^{1} \mathrm{http}: / / \mathrm{lxml} . \mathrm{de}$ 
Thus, a $q \%$ change in $p_{\mathrm{i}}$ leads to a $q \times e_{\mathrm{ik}} \%$ change in $\phi_{\mathrm{k}}$, assuming $q$ is small. Based on this overview, we selected a few model genes for simulating all possible genotypes, using a full factorial design for their associated parameters.

\section{COMPUTER IMPLEMENTATION}

Python code was auto-generated using the CellML code generation service at www.cellml.org. The equations were integrated using the CVODE solver (Cohen and Hindmarsh, 1996), with a Python wrapper for flexible scripting of virtual experiments. Phenotypes were computed with Python and Numpy (Oliphant, 2006). Statistical analyses were done in R 2.10.1 ( $R$ Development Core Team, 2011), using the packages "ggplot2" 0.8.3 (Wickham, 2009) for aggregation and plotting, and "pls" 2.1-0 (Mevik and Wehrens, 2007) for partial least squares (PLS) regression.

\section{RESULTS AND DISCUSSION DESIGN PATTERNS FOR cGP STUDIES}

The workflow in Figure $\mathbf{3}$ exemplifies the design pattern (Wikipedia, 2010) we developed to facilitate the interchange and reuse of its components: the generation of genotypes (e.g., exhaustive enumeration or reduced designs), the mapping of genes to parameters (based on genome databases, e.g., Hancock et al., 2009), physiological models (Le Novere et al., 2006; Lloyd et al., 2008) that map parameters to phenotypes, virtual experiments to generate phenotypes that are defined by the model system's response to some stimulus or perturbation (e.g., voltage clamping, Molleman, 2002), and aggregation from model dynamics to clinically relevant phenotypes (e.g., action potential duration). This pipeline design allows the gluing together of appropriate tools for each task. For instance, experimental designs and statistical analyses were done in R (R Development Core Team, 2011), whereas

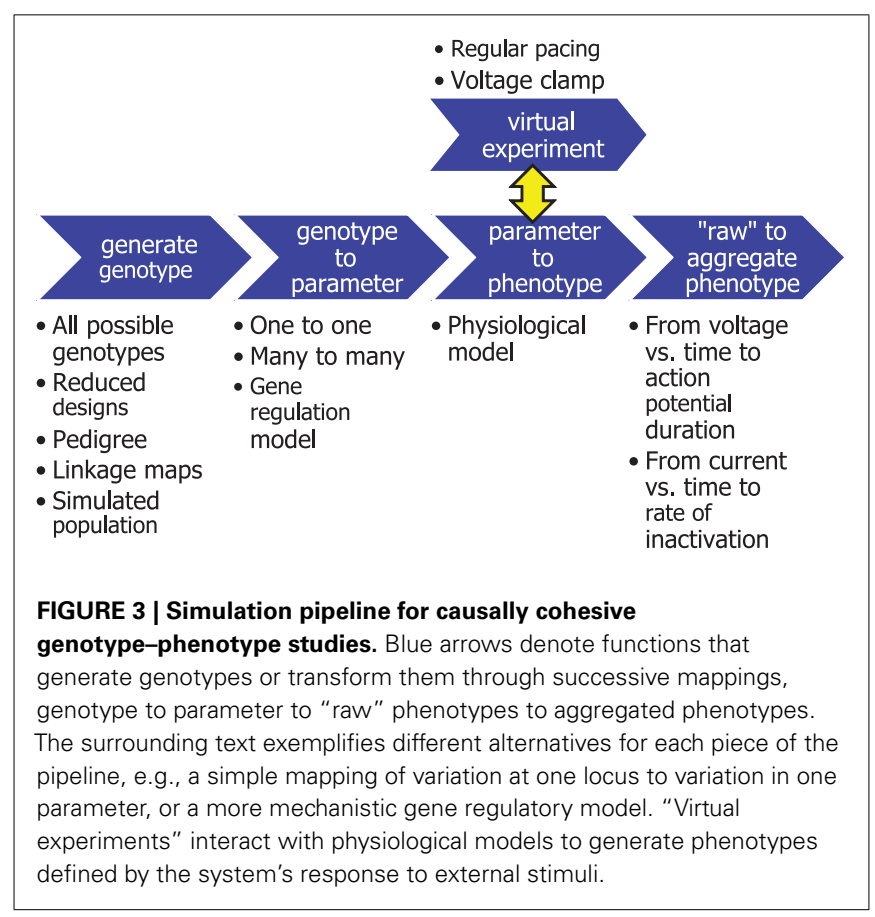

virtual experiments were flexibly described in Python ${ }^{2}$ (see also Langtangen, 2009). The general approach should apply equally well to eventual whole-organ cGP studies.

\section{GENOTYPE-PHENOTYPE ELASTICITIES}

Figure 4 gives a broad overview of the effects of genetic parameter variation on higher-level phenotypes (defined in Figure 2), formulated as elasticities (ratios of relative changes) where applicable. Working with relative changes provides biologically interpretable measures while being dimensionless. For example, the elasticity to Ko (a concentration) of action potential duration to $90 \%$ repolarization (a time) was estimated at 0.45 under pacing at $100-\mathrm{ms}$ intervals. Although this number is dimensionless, we find it helpful to think in terms of "percent per percent." Thus, a 10\% increase in Ko would result in about a 4.5\% increase in APD90. For quantities without an absolute zero, however, relative changes are not meaningful, and sensitivity measures must be expressed using absolute units for either or both of the phenotype and genotypic parameter. For example, the sensitivity of peak voltage to o_Na2 at $100 \mathrm{~ms}$ pacing was $-1.45 \mathrm{mV}$ per $\mathrm{mV}$; the absolute change in peak voltage per relative change in d_Na3 was $-98 \mathrm{mV}$, or $-0.98 \mathrm{mV}$ peak voltage per percent d_Na3; and the relative change in inactivation time $\tau$ per absolute change in o_Na2 was $-3.8 \%$ per $\mathrm{mV}$ (for the fast sodium channel when depolarized to $-30 \mathrm{mV}$ ). In general, the genotype-phenotype elasticity matrix was quite sparse (Figure 4), reflecting a combination of the model's modular structure and whether the simulated genetic effects on parameters were able to penetrate to higher-level phenotypes. A few model components (see Table in Supplementary Material) seemed to have negligible impact on the phenotypes measured, at least locally around the baseline parameter estimate and under the experimental protocols used.

Figure 4 demonstrates the importance of virtual experiments in model validation (see also Cooper et al., 2011). Many effects manifested more clearly in voltage-stepping experiments than under regular pacing, or under fast vs. slow pacing. Thus, genetic parameter variation that would otherwise go unnoticed can be detected by confronting model predictions with experimental data for a range of experimental protocols.

\section{RAW PHENOTYPIC VARIABILITY}

Based on the elasticity analysis above, we selected 10 parameters exemplifying various components and influencing various phenotypes (Figure 1; Table 1), and assumed that each was determined by one biallelic locus, with genotypic values of aa $=50 \%$, $\mathrm{Aa}=100 \%$, and $\mathrm{AA}=150 \%$ of the baseline parameter estimate, for a total of $3^{10}=59049$ parameter scenarios. The assumption of parameter monogenicity is conservative with respect to understanding penetrance and polygenicity, and simplifies the presentation of results while not influencing the major conclusions that follow.

The simulated heart cell dynamics converged without alternans in $56 \%, 62 \%$, and $65 \%$ of cases for pacing intervals of 100,200 ,

\footnotetext{
${ }^{2}$ www.python.org
} 


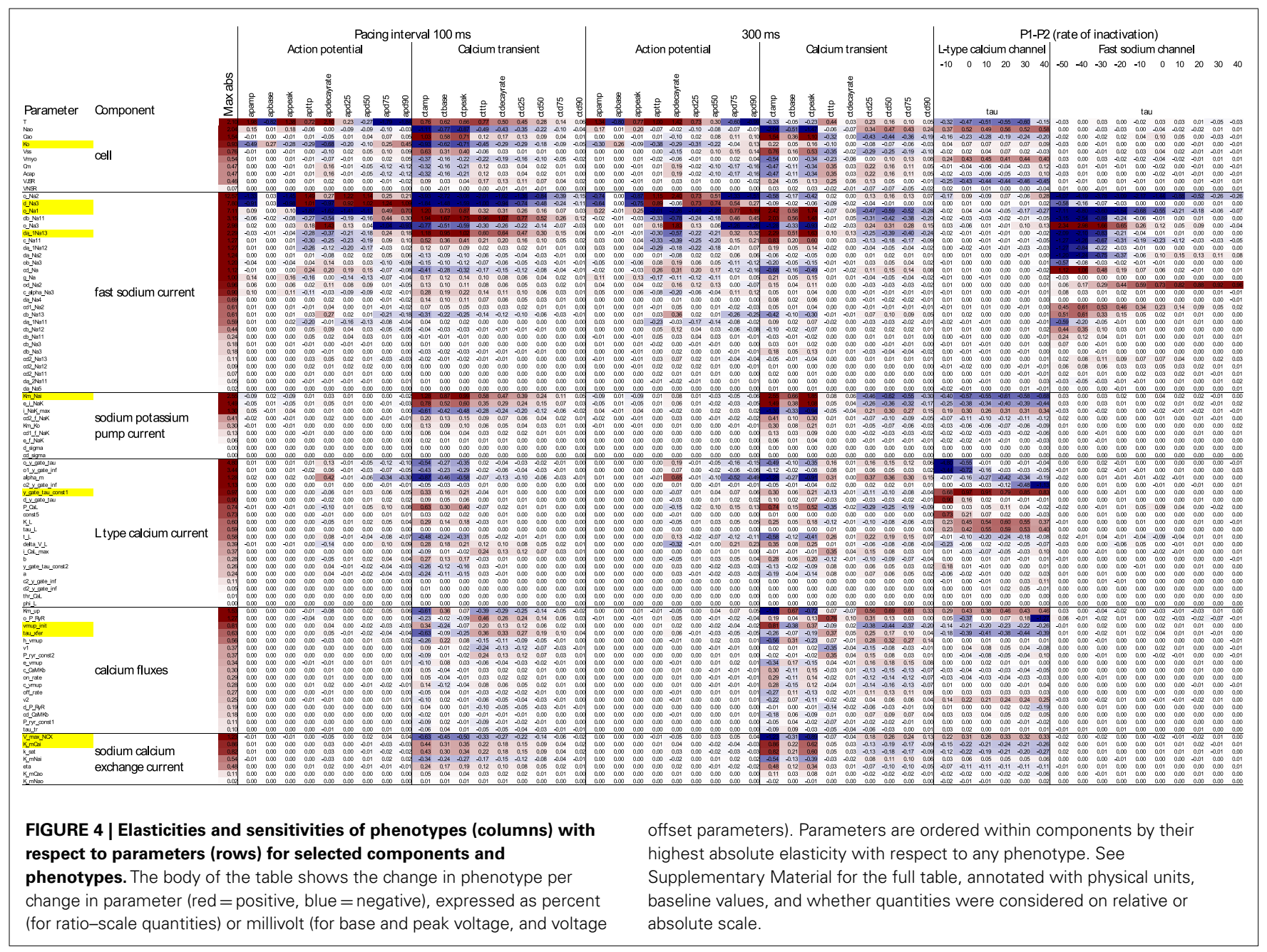

Table 1 | Heart cell model parameters, assumed to reflect individual genes, that were varied $(50,100,150)$ to simulate genetic variation.

\begin{tabular}{llll}
\hline Parameter name & Unit & Baseline value & Description \\
\hline d_Na3 & $\mathrm{mV}$ & 7.7 & Fast Na current, voltage-sensitivity of opening rate (slope) \\
da_1Na13 & $\mathrm{mV}$ & 12 & Fast Na current, voltage-sensitivity of recovery rate from inactivation (slope) \\
O_Na1 & $\mathrm{mV}$ & 2.5 & Fast Na current, voltage-sensitivity of opening rates (offset) \\
Y_gate_tau_const1 & $\mathrm{ms}$ & 8 & L-type Ca current \\
Km_Nai & $\mu \mathrm{M}$ & 16600 & Na-K pump (sensitivity to [Na $\left.{ }^{+}\right]$) \\
tau_xfer & $\mathrm{ms}$ & 8 & Ca fluxes (sensitivity to Ca gradient) \\
vmup_init & $\mu \mathrm{M} / \mathrm{ms}$ & 0.5059 & SERCA, calcium uptake from cytosol to sarcoplasmic reticulum (scales the current) \\
V_max_NCX & $\mathrm{pA} / \mathrm{pF}$ & 3.939 & Na-Ca exchange current (scales the current) \\
K_mCai & $\mu \mathrm{M}$ & 3.6 & Na-Ca exchange current (sensitivity to [Ca2+ $]$ ) \\
Ko & $\mu \mathrm{M}$ & 5400 & Extracellular potassium concentration
\end{tabular}

See Supplementary Material for details on how the parameters enter into the physiological equations.

and $300 \mathrm{~ms}$. The genotypic scenarios that did converge showed considerable phenotypic variation in action potentials, calcium transients, and ion currents under voltage-stepping (Figure 5). Action potentials clustered into a few distinct shapes, whereas the calcium transient showed more continuous phenotypic variation. This may suggest a more polygenic basis for the latter, in that a greater number of processes affect the calcium transient. The voltage-clamp experiment for the baseline scenario showed the usual short-lived current that is cut short by inactivation of ion channels (third panel, red curve in Figure 5). However, in a high proportion of genotypic scenarios the channel failed to inactivate, causing a persistent current throughout the first pulse. 

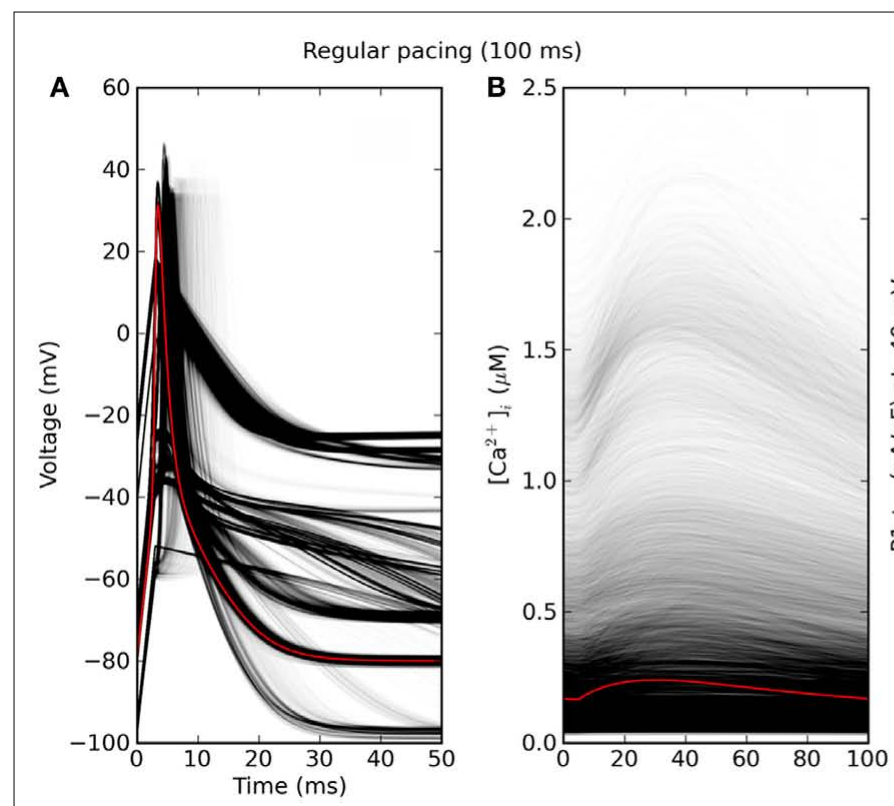

FIGURE 5 | Phenotypic variability simulated by varying in silico genes that each determine one model parameter. Red is the baseline genotype. (A) Action potential and (B) calcium transient under regular pacing at $100 \mathrm{~ms}$ intervals. Only genotypes

As a consequence, the aggregate phenotype of a time-scale of inactivation $(\tau)$ was not well defined in these cases. In summary, a cGP model generates phenotypic data that can be directly confronted with empirical measurements, giving a causal account of genetic concepts such as penetrance, dominance, and epistasis. Below we exemplify how the data can be aggregated for purposes of analysis and interpretation.

\section{PHENOTYPIC CORRELATIONS}

Scatterplots are useful in visualizing the covariation between pairs of scalar phenotypes (cf. Figure 2) that results from simulated genetic variation (Figure 6). The distinct AP shapes in Figure 5 are reflected in the strong phenotypic correlation between APD25 and APD90 within distinct groups (Figure 6, upper red highlight). Variation in calcium transient phenotypes was more continuous, though often quite irregular (middle and lower red highlights).

Clustering in phenotypic values may suggest that one or a few genes underlie the variation. Coloring points by genotype is informative in simple cases, for example AP peak and base vs. Ko (Figure 6, inset). However, when phenotypic ranges overlap, and multiple genotypic or other causal variation is involved, multivariate methods can give a better overview of many dependencies simultaneously, as shown below.

\section{CHARACTERIZING A HIGH-DIMENSIONAL GENOTYPE-TO-PHENOTYPE MAP}

Partial least squares regression (Martens and Næs, 1992) provides a low-dimensional approximation of the covariance between responses (here phenotypes) and predictors (here genotypic parameters). PLS compresses both the predictors and responses into

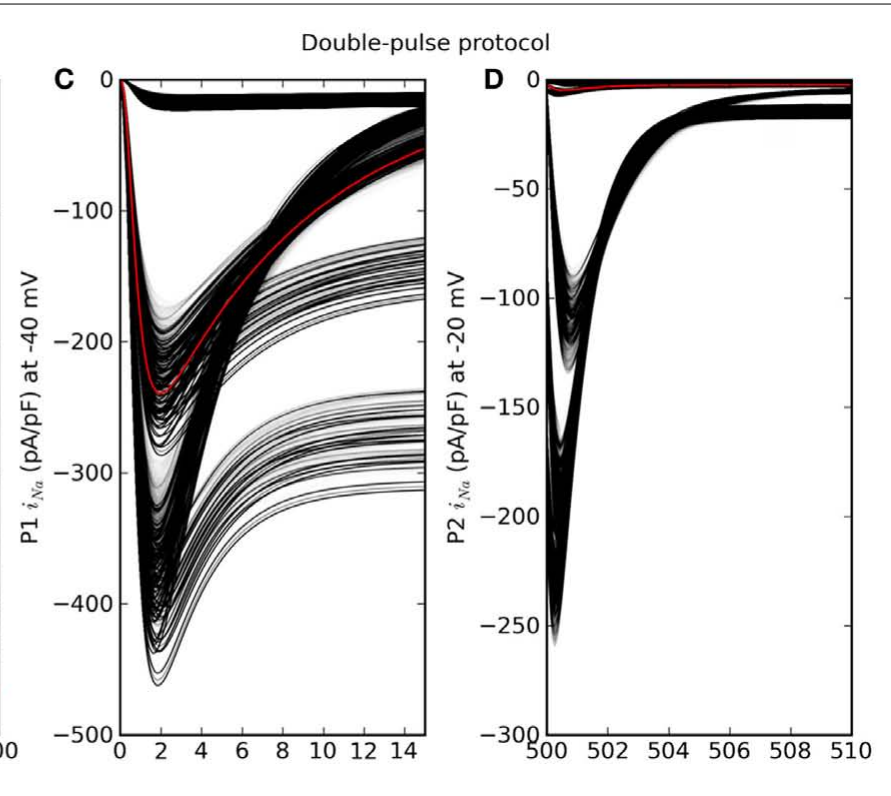

for which dynamics converged without alternans are shown (about $56 \%$ of $3^{10}=59049$ cases). (C,D) Fast sodium current under a double-pulse voltage-clamp experiment. Note that the time axes are truncated.

their most relevant subspaces, spanned by a basis of covariance eigenvectors (weighting each original variable by so-called loadings; scores denotes each observation's coordinates in the new basis). The correlation between the original variables and the scores are called correlation loadings. Thus, Figure 7 places simulated loci and phenotypic measures onto a few common axes, concisely depicting their patterns of covariation.

The placement of base voltage and other action potential phenotypes at the extremes of the PC1 axis (correlation loadings for PLS component 1) shows that these enter strongly into the first major component of phenotypic variation, as does the time-scale of the i_CaL current. The second component brings in calcium transient phenotypes. Together, the first pair of phenotypic components account almost fully (outer circle) for variation in the base voltage, whereas the variance in the calcium transient is mostly relegated to later components, in particular the combination of PC2 and PC3. The proximity of, e.g., APD25 and AP time to peak shows them to be highly correlated, whereas their being at right angles to tau_i_CaL means that these groups of phenotypes are fairly uncorrelated in this two-dimensional projection of the data. AP base is strongly negatively correlated with the other AP phenotypes, as evident from its placement opposite the others. The serial, but weakening correlation between recovery times is evident from their placement along a curve in the diagram, for both the action potential and calcium transient.

The first genotypic PLS component is dominated by Ko and y_gate_tau_const1, which are strongly correlated with the phenotypes AP base and tau_i_CaL, respectively. In summary, the PLS analysis gives a very concise depiction of the genotypeto-phenotype map, reflecting findings of both the bivariate scatterplots and variance decompositions (below). 


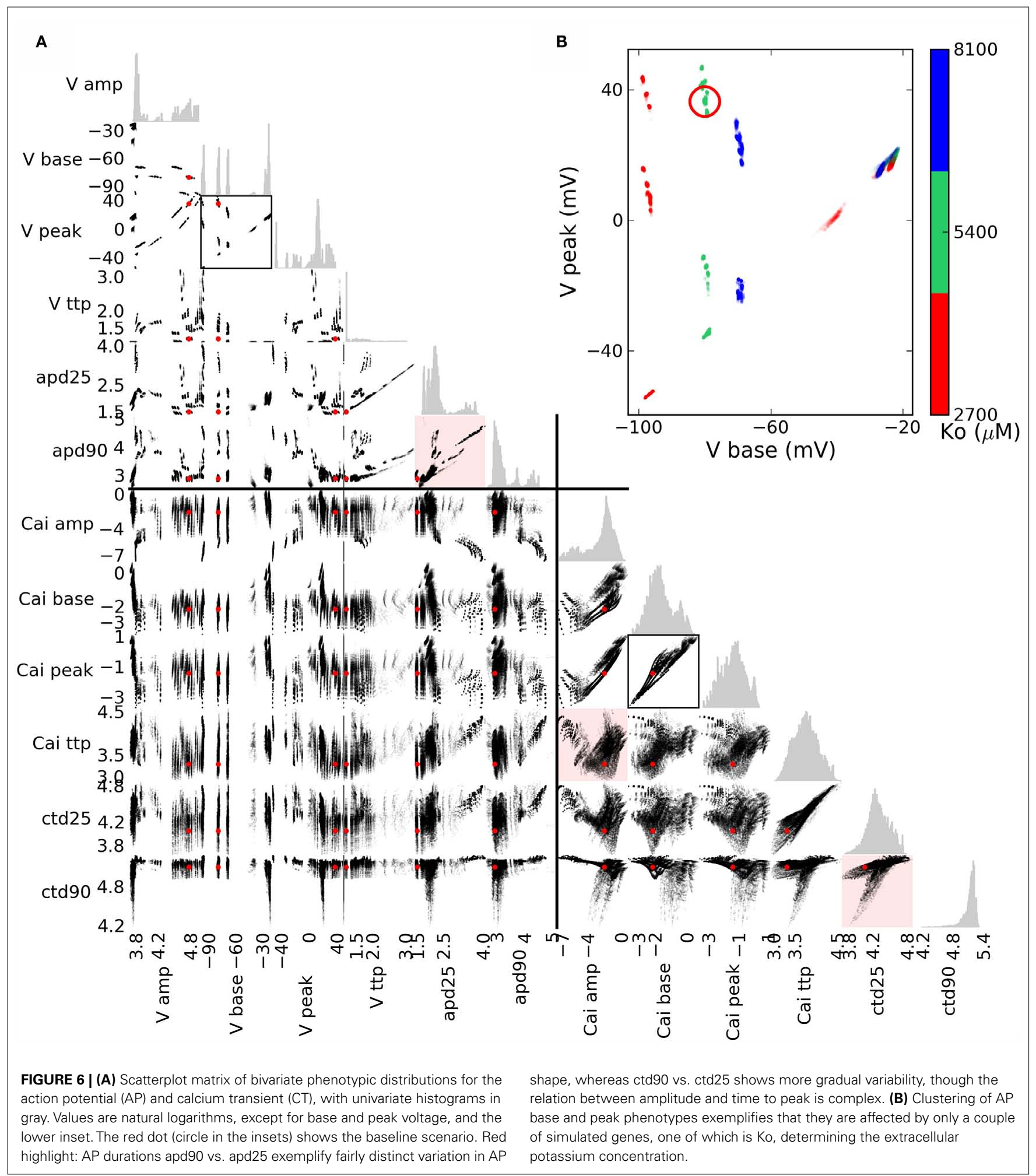

For cases where interesting patterns apply only to subsets of the data, clustering-based methods may offer an alternative to specifying interaction terms parametrically (Tøndel et al., 2011). Many phenotypes may apply only in a portion of cases; for instance, action potential duration is well defined only if heart cell dynamics converges to stable dynamics without alternans. Such cases are amenable to a combined approach, quantifying the continuous phenotypic variation for the cases where it is well defined (Figures 7 and 9), and exploring the causes of failure using, e.g., logistic regression (Hosmer and Lemeshow, 2000). 


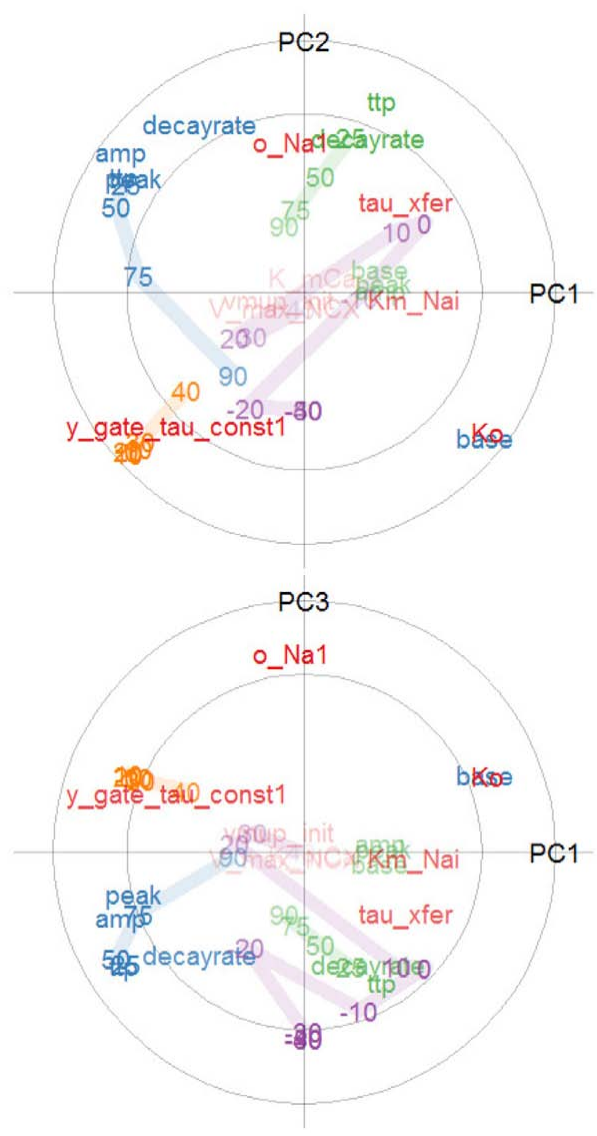

FIGURE 7 | Overview of a multivariate genotype-phenotype map: Correlation loading plot matrix of the first three components of a partial least squares regression of action potential, calcium transient, and voltage-clamp phenotypes (categorized by color) against genotypic variables (red). The axes show correlations between a PLS component and each genotypic or phenotypic variable. The circles indicate 50 and $100 \%$ a Locus

a ap

a $\mathrm{ct}$

a tau_i_Na

a tau_i_CaL

\section{THE GENETIC BASIS OF AN IN SILICO DISEASE PHENOTYPE}

We believe that defining disease phenotypes in silico holds great potential for improving understanding of different proximate causes underlying medical signs and symptoms, and of the interactions between genetic and environmental parameters that underlie epistasis and incomplete penetrance. In our study, simulated genetic variation had strong effects on the viability of in silico heart cells, as measured by their calcium handling (Figure 8). Among the genotypic scenarios that did converge, increasing the parameter d_Na3 ran the risk of reducing peak calcium to an unviable level, while reducing the voltage-sensitivity could maintain base calcium so high as to prevent proper relaxation (Figure 8). However, model dynamics failed to even converge in almost all cases for certain combinations of the genes for da_1Na13 and d_Na3, which modify the fast sodium channel's voltage-dependency of rates of opening and recovery from inactivation, respectively (Figure 1; Table 1). The epistatic interactions between these simulated genes were not simple; gene substitutions for d_Na3 that were harmful alone could compensate for problems arising from substitutions of da_1Na13
(Figure 8), or only had an impact under stress such as fast pacing (Figure 4).

Although simple, this example points to the possibility of classifying diseased and healthy individuals based on clinically relevant phenotypic measures, while obtaining more refined insight by analyzing the high-dimensional phenotypic variation underlying the binary classification. Highly complex interactions between genetic factors and environmental challenges may be a generic feature of complex diseases, in which case cGP models can shed light on the interplay between genetic, age-related, and lifestyle factors, based on how disease manifests at multiple phenotypic levels in a causally cohesive model.

\section{VARIANCE DECOMPOSITION}

Traditional variance decompositions can provide helpful indications of how phenotypic variability arises from genotypic and other variation in parameters. In our study, the analysis was complicated by the fact that AP and CT phenotypes were not well defined in cases of alternans (Figure 8). For simplicity, we contrasted variance decompositions for two well-behaved subsets of 

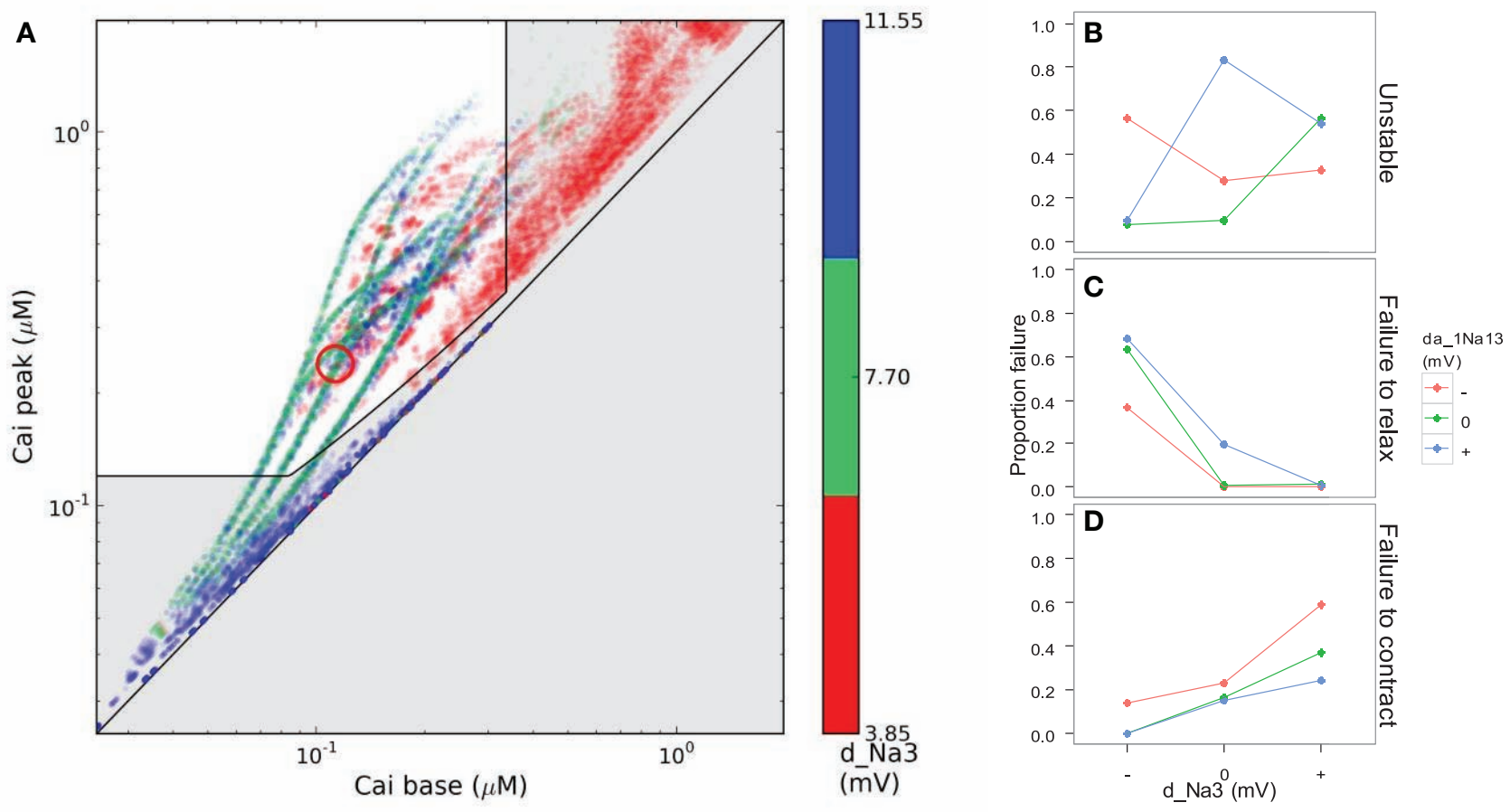

FIGURE 8 | Simulated disease phenotype based on the calcium transient, a proxy for muscle contraction, in scenarios with stable dynamics at $\mathbf{2 0 0} \mathbf{~ m s}$ pacing. (A) The gray area shows failure to contract (bottom), failure to relax (right), or insufficient calcium transient amplitude (near diagonal). Color indicates levels of $d \_N a 3$, the voltage-sensitivity of the opening rate of the fast sodium channel. (B) Interaction of d_Na3 and da_1Na13 (voltage-sensitivity of the rate of recovery from inactivation) with respect to the proportion of simulated genotypes that converged to stable dynamics. The middle and bottom panels show the proportion of stable scenarios suffering from excessive base levels of cytosolic calcium (C) or insufficient peak calcium (D). the data, namely baseline and low d_Na3 at baseline values of da_1Na13. We fitted linear regression models (function $\mathrm{lm}$ in R) for each scalar measure of the action potential and calcium transient, with genotypic variables as predictors, including secondorder interactions and quadratic terms. In the resulting variance decompositions (Figure 9), phenotypes ranged from being monogenic to oligogenic to polygenic, even under the conservative assumption that low-level parameters were strictly monogenic.

Figure 9 illustrates the potential of cGP models in systematically assessing the degree to which the -genicity of complex traits and associated penetrance patterns are likely to change as a function of the genetic background. Action potential phenotypes appeared largely monogenic (due to Ko, with very non-additive effects, e.g., on APD90) at baseline d_Na3, but di- or trigenic at low d_Na3. The AP amplitude and early-stage AP duration varied by Km_Nai (in the sodium-potassium pump component), whereas AP durations were also influenced by y_gate_tau_const1 (in the L-type calcium current component). (AP time to peak had zero variability at low d_Na3, because repolarization was already underway by the end of the stimulus.) The two backgrounds showed also very different major determinants of calcium transient phenotypes. Under baseline d_Na3, the greatest variance component was due to tau_xfer (affecting calcium release from subspace to cytosol), whereas vmup_init (affecting calcium re-uptake from cytosol) dominated at low d_Na3. These results suggest that varying-genicity and penetrance may be generic features of complex physiological traits, and that these features can be systematically and meaningfully studied by use of cGP models.

For example, findings of quantitative trait loci (QTLs) underlying complex traits are often not consistent across populations (Beavis, 1998). cGP models may shed light on whether QTLs for variation in lower-level processes are likely to manifest in higher-level phenotypes and to assess the associated penetrance characteristics, informing the interpretation of empirical data and guiding experimental search for putative QTLs.

\section{PARAMETERS ARE PHENOTYPES}

From the assumption that low-level parameters were strictly monogenic, emerged a polygenic basis for phenotypes such as the characteristic time-scale of ion channels; phenotypes that might be used as parameters in more aggregate models (Rajasingh et al., 2008). Similarly, many parameters that we assumed monogenic and constant could instead be derived from mechanistic submodels. For instance, the output of gene regulatory models for the expression levels of ion transport proteins corresponds directly to model parameters that scale ion currents, such as V_max_NCX or vmup_init in Table 1 (see, e.g., Gjuvsland et al., 2006) An example of gene regulatory responses was seen with conditional knockout of the SERCA channel, which was partially compensated for by increased expression of other calcium channels (Andersson et al., 


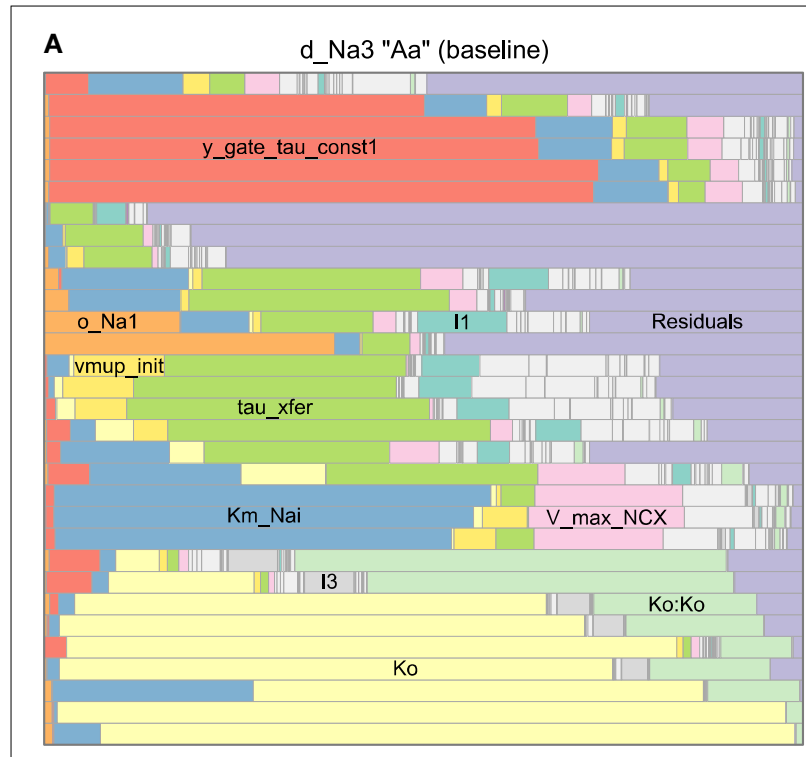

FIGURE 9 | Effect of a hypothetical gene (d_Na3) on variance decompositions of action potential (AP), calcium transient (CT), and voltage-clamp (VT) phenotypes, based on linear regression against genotypic parameters including second-order and quadratic terms to capture genetic interactions and intralocus non-additivity.

Simulated genes with large variance components are labeled; Ko:Ko denotes the quadratic term for Ko. Second-order interactions were minor in this case except those of tau_xfer and Km_Nai (labeled I1), tau_xfer
B

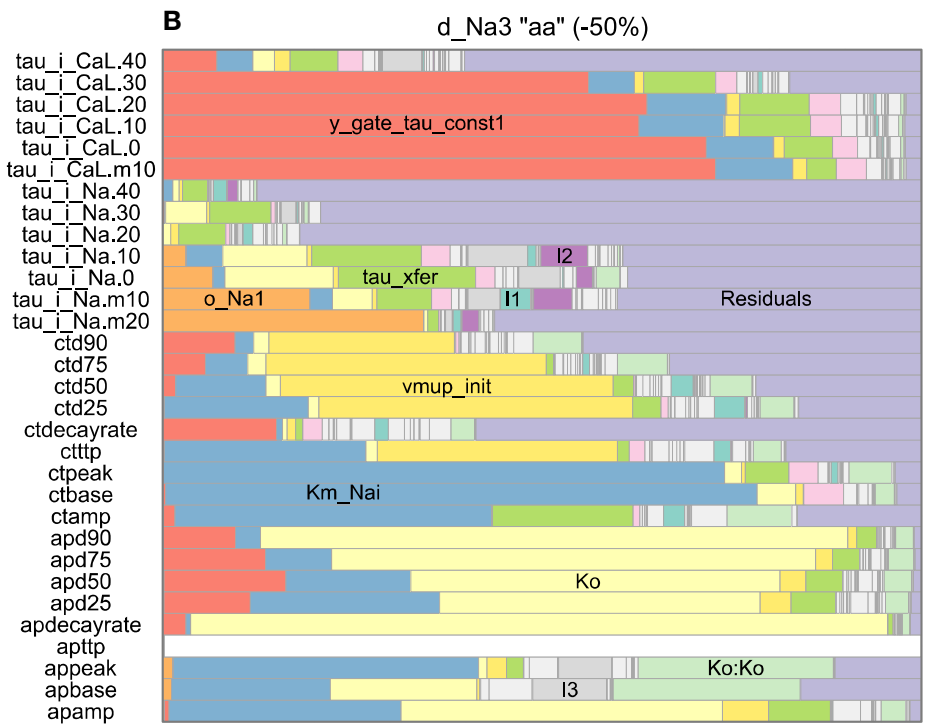

and Ko (I2), Km_Nai and Ko (I3), or tau_xfer and vmup_init (I4). Higher-order terms are lumped in "Residuals." Voltage-clamp phenotypes include the characteristic time-scale (tau) for the L-type calcium and fast sodium currents after a step depolarization (e.g., to $-50 \mathrm{mV}$ for tau_i_Na.m50). To restrict the illustration to well-behaved subsets of the data, only scenarios with baseline $(\mathbf{A})$ or low $(\mathbf{B})$ levels of d_Na3, baseline levels of da_1Na13, and a stimulus period of $200 \mathrm{~ms}$ (for AP and CT) are shown.
2009). Modeling signal transduction and gene regulation (Cooling et al., 2009), electromechanical coupling (Niederer and Smith, 2007) and whole-organ phenomena (Nordsletten et al., 2011) are further promising targets for realistic gene-to-parameter mapping in cGP modeling. The requisite data and tools are just becoming available through databases, coding standards, and ontologies such as those promoted by the Physiome Project (Hunter and Borg, 2003) and the Virtual Physiological Human (Hunter et al., 2010). For example, the knowledge in genomic and phenomic databases can become vastly more usable through annotation with biologically meaningful, yet machine-processable descriptors. Phenotypic assays can be linked to models by complementing model repositories (Le Novere et al., 2006; Lloyd et al., 2008) with simulation experiment descriptions in appropriate languages (Köhn and Novère, 2011).

\section{CONCLUDING REMARKS}

In their commentary entitled "Life after GWA studies," Dermitzakis and Clark (2009) conclude that "A major breakthrough will be to predict and interpret the effect of mutational and biochemical changes in human cells and understand how this signal is transmitted spatially (among tissues) and temporally (spanning development)." Causally cGP modeling addresses exactly this vision by bridging the gap between genomic information and the high-dimensional phenotypes of individuals. The physiologically validated cell model in our case study exhibits many wellknown genetic phenomena such as variable penetrance of a binary disease phenotype, intralocus dominance, non-linear responses, interlocus epistasis, varying degrees of phenotypic correlation
(Figure 6), and a range from monogenic to oligo- and polygenic traits (Figure 9). Thus, model results are amenable both to standard quantitative genetic methods (Lynch and Walsh, 1998) and novel applications of high-dimensional data analysis (Martens and Næs, 1992). The close parallel between empirical and cGP studies makes for a tight link to experimental work (cf. Figure 3). Whereas passive observation may not provide the most informative phenotypes, experiments (real and virtual) can be designed to bring into play system components whose importance manifests only under certain conditions or perturbations. Because the parameter-to-phenotype model is based on physiological principles and empirical data (Hunter and Borg, 2003), a cGP study generates experimentally verifiable hypotheses for both physiological and genetic studies at multiple phenotypic levels (Rajasingh et al., 2008) in a way that statistical-genetic studies cannot (Dermitzakis and Clark, 2009). In personalized medicine, this approach can lead toward a systemic understanding of what it takes to force a diseased system into a healthier state. Incorporating the effects of environmental and lifestyle variation on parameters and phenotypes is an important next step. A computational physiological genomics will have to involve a whole range of theoretical methodologies and approaches, but we find it hard to envisage how we can achieve a deep understanding of the genotype-phenotype relationship without letting cGP modeling become a key element in this emerging discipline.

\section{ACKNOWLEDGMENTS}

This work was funded by the Research Council of Norway under the eVITA programme, project number 178901/V30. NOTUR, 
the Norwegian metacenter for computational science, provided computing resources under project nn4653k. We are grateful to Johan Hake for help in getting acquainted with the Bondarenko model.

\section{REFERENCES}

Andersson, K. B., Birkeland, J. A. K., Finsen, A. V., Louch, W. E., Sjaastad, I., Wang, Y., Chen, J., Molkentin, J. D., Chien, K. R., Sejersted, O. M., and Christensen, G. (2009). Moderate heart dysfunction in mice with inducible cardiomyocyte-specific excision of the Serca2 gene. J. Mol. Cell. Cardiol. 47, 180-187.

Bateson, W. (1906). "The progress of genetic research: an inaugural address to the third conference on hybridisation and plant-breeding," in Scientific Papers of William Bateson (1928), ed. R. S. Punett (Cambridge: University Press), 142-151.

Beavis, W. D. (1998). "QTL analyses: power, precision, and accuracy," in Molecular Dissection of Complex Traits, ed. A. H. Paterson (Boca Raton, FL: CRC Press), 145-162.

Bondarenko, V. E., Szigeti, G. P., Bett, G. C. L., Kim, S.-J., and Rasmusson, R. L. (2004). Computer model of action potential of mouse ventricular myocytes. Am. J. Physiol. Heart Circ. Physiol. 287, H1378-H1403.

Burns, J. (1970). "The synthetic problem and the genotype-phenotype relation in cellular metabolism," in Towards a Theoretical Biology. 3. Drafts. An I.U.B.S. Symposium, ed. C. H. Waddington (Chicago: Aldine Publishing Company), 47-51.

Cohen, S. D., and Hindmarsh, A. C. (1996). CVODE, a stiff/nonstiff ODE solver in C. Comput. Phys. 10, 138-143.

Cooling, M. T., Hunter, P., and Crampin, E. J. (2009). Sensitivity of NFAT cycling to cytosolic calcium concentration: implications for hypertrophic signals in cardiac myocytes. Biophys. J. 96, 2095-2104.

Cooper, J., Mirams, G. R., and Niederer, S. A. (2011). High-throughput functional curation of cellular electrophysiology models. Prog. Biophys. Mol. Biol. 107, 11-20.

Dermitzakis, E. T., and Clark, A. G. (2009). Life after GWA studies. Science 326, 239-240.

Falconer, D. S., and Mackay, T. F. C. (1996). Introduction to Quantitative Genetics. 4th Edn. Harlow: Longman Group.

Gilchrist, M. A., and Nijhout, H. F. (2001). Nonlinear developmental processes as sources of dominance. Genetics 159, 423-432.
Gjuvsland, A., Hayes, B., Meuwissen, T., Plahte, E., and Omholt, S. (2007a). Nonlinear regulation enhances the phenotypic expression of trans-acting genetic polymorphisms. BMC Syst. Biol. 1, 32. doi:10.1186/1752-0509-1-32

Gjuvsland, A., Plahte, E., and Omholt, S. (2007b). Thresholddominated regulation hides genetic variation in gene expression networks. BMC Syst. Biol. 1, 57. doi:10.1186/1752-0509-1-57

Gjuvsland, A. B., Hayes, B. J., Omholt, S. W., and Carlborg, O. (2007c). Statistical epistasis is a generic feature of gene regulatory networks. Genetics $175,411-420$.

Gjuvsland, A. B., Hayes, B. J., Omholt, S. W., and Carlborg, O. (2006). Statistical epistasis is a generic feature of gene regulatory networks. Genetics 175, 411-420.

Gjuvsland, A. B., Plahte, E., Ådnøy, T., and Omholt, S. W. (2010). Allele interaction - single locus genetics meets regulatory biology. PLoS ONE 5, e9379. doi:10.1371/journal.pone.0009379

Hancock, J. M., Mallon, A.-M., Beck, T., Gkoutos, G. V., Mungall, C., and Schofield, P. N. (2009). Mouse, man, and meaning: bridging the semantics of mouse phenotype and human disease. Mamm. Genome 20, 457-461.

Hosmer, D. W., and Lemeshow, S. (2000). Applied logistic Regression, 2nd Edn. New York: Wiley.

Hund, T. J., Kucera, J. P., Otani, N. F., and Rudy, Y. (2001). Ionic charge conservation and long-term steady state in the Luo-Rudy dynamic cell model. Biophys. J. 81, 3324-3331.

Hunter, P., Coveney, P. V., de Bono, B., Diaz, V., Fenner, J., Frangi, A. F., Harris, P., Hose, R., Kohl, P., Lawford, P., McCormack, K., Mendes, M., Omholt, S., Quarteroni, A., Skår, J., Tegner, J., Randall Thomas, S., Tollis, I., Tsamardinos, I., van Beek, J. H. G. M., and Viceconti, M. (2010). A vision and strategy for the virtual physiological human in 2010 and beyond. Philos. Transact. A Math. Phys. Eng. Sci. 368, 2595-2614.

Hunter, P. J., and Borg, T. K. (2003). Integration from proteins to organs: the physiome project. Nat. Rev. Mol. Cell Biol. 4, 237-243.

Köhn, D., and Novère, N. (2011). "SED-ML - an XML format for the implementation of the MIASE

\section{SUPPLEMENTARY MATERIAL}

The Supplementary Material for this article can be found online at http://www.frontiersin.org/Genomic_Physiology_/10.3389/fphys. 2011.00106/abstract

guidelines [online]," in Computational Methods in Systems Biology, eds M. Heiner and A. M. Uhrmacher (Berlin: Springer Berlin Heidelberg), 176-190.

Langtangen, H. P. (2009). Python Scripting for Computational Science, 3rd Edn. Berlin: Springer Berlin Heidelberg.

Le Novere, N., Bornstein, B., Broicher, A., Courtot, M., Donizelli, M., Dharuri, H., Li, L., Sauro, H., Schilstra, M., Shapiro, B., Snoep, J. L., and Hucka, M. (2006). BioModels database: a free, centralized database of curated, published, quantitative kinetic models of biochemical and cellular systems. Nucl. Acids Res. 34 D689-D691.

Li, L., Niederer, S. A., Idigo, W. Zhang, Y. H., Swietach, P., Casadei, B., and Smith, N. P. (2010). A mathematical model of the murine ventricular myocyte: a datadriven biophysically based approach applied to mice overexpressing the canine NCX isoform. Am. J. Physiol. Heart Circ. Physiol. 299, H1045-H1063.

Lloyd, C. M., Halstead, M. D., and Nielsen, P. F. (2004). CellML: its future, present and past. Prog. Biophys. Mol. Biol. 85, 433-450.

Lloyd, C. M., Lawson, J. R., Hunter, P. J., and Nielsen, P. F. (2008). The cellML model repository. Bioinformatics 24 , 2122-2123.

Lynch, M., and Walsh, B. (1998). Genetics and Analysis of Quantitative Traits, 1st Edn. Sunderland: Sinauer Associates.

Martens, H., and Næs, T. (1992). Multivariate Calibration. 1st Edn. Chichester: Wiley.

Martens, H., Veflingstad, S., Plahte, E., Martens, M., Bertrand, D., and Omholt, S. (2009). The genotypephenotype relationship in multicellular pattern-generating models - the neglected role of pattern descriptors. BMC Syst. Biol. 3, 87. doi:10.1186/1752-0509-3-87

Mevik, B. H., and Wehrens, R. (2007). The pls package: principal component and partial least squares regression in R. J. Stat. Softw. 18, 1-24.

Molleman, A. (2002). Patch Clamping: An Introductory Guide to Patch Clamp Electrophysiology, 1st Edn. New York, NY: Wiley.

Niederer, S. A., Fink, M., Noble, D., and Smith, N. P. (2008). A metaanalysis of cardiac electrophysiology computational models. Exp. Physiol. 94, 486-495.

Niederer, S. A., and Smith, N. P. (2007). An improved numerical method for strong coupling of excitation and contraction models in the heart. Prog. Biophys. Mol. Biol. 96, 90-111.

Noble, D. (2002a). Modeling the heart from genes to cells to the whole organ. Science 295, 1678-1682.

Noble, D. (2002b). The rise of computational biology. Nat. Rev. Mol. Cell Biol. 3, 459-463.

Nordsletten, D. A., Niederer, S. A., Nash, M. P., Hunter, P. J., and Smith, N. P. (2011). Coupling multi-physics models to cardiac mechanics. Prog. Biophys. Mol. Biol. 104, 77-88.

Oliphant, T. E. (2006). Guide to NumPy. Spanish Fork, UT: Trelgol Publishing.

Omholt, S. W., Plahte, E., Oyehaug, L., and Xiang, K. (2000). Gene regulatory networks generating the phenomena of additivity, dominance and epistasis. Genetics 155, 969-980.

Peccoud, J., Velden, K. V., Podlich, D., Winkler, C., Arthur, L., and Cooper, M. (2004). The selective values of alleles in a molecular network model are context dependent. Genetics 166, 1715-1725.

R Development Core Team. (2011). R: A language and environment for statistical computing. Vienna: Foundation for Statistical Computing.

Rajasingh, H., Gjuvsland, A. B., Våge, D. I., and Omholt, S. W. (2008). When parameters in dynamic models become phenotypes: a case study on flesh pigmentation in the chinook salmon (Oncorhynchus tshawytscha). Genetics 179, 1113-1118.

Roberts, R., and Brugada, R. (2003). Genetics and Arrhythmias. Annu. Rev. Med. 54, 257-267.

Roepke, T. K., and Abbott, G. W. (2006). Pharmacogenetics and cardiac ion channels. Vascul. Pharmacol. 44, 90-106.

Sanguinetti, M. C., and Tristani-Firouzi, M. (2006). hERG potassium channels and cardiac arrhythmia. Nature 440, 463-469.

Tøndel, K., Indahl, U., Gjuvsland, A. B., Vik, J. O., Hunter, P. J., Omholt, S. W., and Martens, H. (2011). Hierarchical Cluster-Based Partial Least Squares Regression (HC-PLSR) is an efficient tool for metamodelling of nonlinear 
dynamic models. BMC Syst. Biol. 5, 90ff. doi:10.1186/1752-0509-5-90

Welch, S. M., Dong, Z. S., Roe, J. L., and Das, S. (2005). Flowering time control: gene network modelling and the link to quantitative genetics. Aust. J. Agric. Res. 56, 919-936.

Wickham, H. (2009). ggplot2: Elegant Graphics for Data Analysis, 1st Edn. New York, NY: Springer.

Wikipedia. (2010). Design pattern (computer science) [Online]. Wikipedia,
The Free Encyclopedia Wikimedia Foundation. Available at: http:// en.wikipedia.org/w/index.php?title =Design_pattern_(computer_scien ce) $\&$ oldid $=378971385$ [accessed 17 August 2010].

Conflict of Interest Statement: The authors declare that the research was conducted in the absence of any commercial or financial relationships that could be construed as a potential conflict of interest.

Received: 08 September 2011; accepted: 05 December 2011; published online: 28 December 2011.

Citation: Vik JO, Gjuvsland AB, Li L, Tondel K, Niederer S, Smith NP, Hunter PJ and Omholt SW (2011) Genotypephenotype map characteristics of an in silico heart cell. Front. Physio. 2:106. doi: 10.3389/fphys.2011.00106
This article was submitted to Frontiers in Genomic Physiology, a specialty of Frontiers in Physiology.

Copyright $\odot 2011$ Vik, Gjuvsland, Li, Tøndel, Niederer, Smith, Hunter and Omholt. This is an open-access article distributed under the terms of the Creative Commons Attribution Non Commercial License, which permits noncommercial use, distribution, and reproduction in other forums, provided the original authors and source are credited. 